\title{
No es tronco todo lo que reluce: a propósito de un caso
}

\author{
Enrique Sánchez Muñoz \\ Javier Borrego Rodríguez \\ Tomás Benito González \\ Ignacio Iglesias Garriz \\ Felipe Fernández Vázquez
}

Servicio de Cardiología Complejo Universitario de León. España

Recibido: : 20/12/2020

Aceptado: 31/01/2021

En línea: 30/04/2021

\section{Correspondencia}

Javier Borrego Rodríguez

jborrego458@gmail.com

Citar como: Sánchez Muñoz E, Borrego Rodríguez J, Benito González T, Iglesias Gárriz I, Fernández Vázquez F. No es tronco todo lo que reluce: A propósito de un caso. Rev Ecocar Pract (RETIC). 2021 (Abril); 4 (1): 4-7. doi: 10.37615/retic.v4n1a4.

Cite this as: Sánchez Muñoz E, Borrego Rodríguez J, Benito González T, Iglesias Gárriz I, Fernández Vázquez F. All that glitters is not a trunk: Case report. Rev Ecocar Pract (RETIC). 2021 (Abril); 4 (1): 4-7. doi: 10.37615/retic.v4n1a4.

\section{Palabras clave}

$\triangleright$ Síndrome aórtico agudo

$\triangleright$ Disección aórtica

$\triangleright$ Flap Aórtico

\section{RESUMEN}

El Síndrome Aórtico Agudo (SAA) es una situación clínica que engloba varias entidades, todas ellas caracterizadas por una presentación clínica común, que consiste en la extravasación de sangre fuera de la verdadera luz aórtica. El SAA más frecuente (80\%) es la disección aórtica, producida como consecuencia de un desgarro intimal, y típicamente localizada en la aorta torácica ascendente. Esta entidad presenta una mortalidad cercana al 40-50\%.

Se muestra el caso de un paciente con disección de aorta ascendente muy ilustrativo tanto desde el punto de vista de la imagen cardíaca como desde la clínica y la sintomatología con la que se presentó en el Servicio de Urgencias Hospitalarias.

\section{Keywords}

$\triangleright$ Acute aortic syndrome

$\triangleright$ Aortic dissection

$\triangleright$ Aortic flap

\section{ABSTRACT}

Acute Aortic Syndrome (AAS) is a clinical situation including several entities, all of them characterized by a common clinical presentation, the extravasation of blood outside the true aortic lumen. The most common AAS (80\%) variant is Aortic Dissection, produced because of an intimal tear and typically located in the ascending thoracic aorta. This entity presents a mortality close to 40-50\%.

We hereby present the case of a patient with a very illustrative ascending aortic dissection, both from the cardiac image and clinical the points of view.

\section{Presentación del caso}

Paciente de 71 años que acude al Servicio de Urgencias Hospitalarias (SUH) por dolor torácico. En su historia cardiológica destacaba hipertensión arterial de difícil control pese a tres fármacos antihipertensivos y una insuficiencia cardíaca con fracción de eyección en rango intermedio (último ecocardiograma con FEVI 48\%, parcialmente recuperada desde 2011), secundaria a una miocardiopatía dilatada de etiología no isquémica. Actualmente se encontraba en clase funcional I-II NYHA. Además, era seguido en consultas externas de cardiología por una dilatación aneurismática de la aorta torácica ascendente $(49 \mathrm{~mm})$ sin datos de progresión en los últimos 3 años. Como otros antecedentes había tenido 7 meses antes una rotura de un aneurisma de la arteria ilíaca común izquierda que se trató con reparación endovascular aorto-monoiliaco derecho y bypass femoro-femoral derecha-izquierda con prótesis de Dacron.

A su llegada al SUH, el paciente impresiona de mal estado general, y refiere un dolor súbito centrotorácico transfixivo irradiado a espalda de 2 horas de evolución asociado a con cortejo vegetativo intenso. Siguiendo el protocolo de dolor torácico de nuestro centro, se realiza un electrocardiograma (ECG) en los primeros 10 minutos tras su llegada (Figura 1), que sugiere Síndrome Coronario Agudo con enfermedad de tronco coronario izquierdo (TCI), por lo que se traslada al paciente al Box de Críticos, y se avisa a los cardiólogos de guardia.

A la exploración física presenta hipoperfusión con frialdad y palidez en la extremidad superior izquierda, donde no se consiguen obtener cifras de tensión 
arterial con el esfingomanómetro. La tensión arterial medida en la extremidad superior derecha era $70 / 45 \mathrm{~mm} \mathrm{Hg}$.

En la auscultación cardíaca destaca un soplo diastólico III/IV en foco aórtico. Los pulsos en extremidades inferiores eran débiles pero conservados. SatO $88 \%$.

A nuestra llegada ya se le ha realizado una radiografía de tórax (Figura 2), una gasometría (donde destaca un pH de 7.33, y un láctico de $2.8 \mathrm{mmol} / \mathrm{L}$ ), una analítica sanguínea con marcadores de daño miocárdico (Troponina UItrasensible de 18 [Rango 0-13], un Dímero D de 6.789, y un NT-proBNP de $1.293 \mathrm{pg} / \mathrm{ml}$ ) y un nuevo electrocardiograma donde se visualiza normalización del segmento ST.

Ante la inestabilidad hemodinámica del paciente -en situación de shock-, la exploración física que presentaba, y los hallazgos del ECG, se realiza un ecocardiograma con un equipo de bolsillo (Vídeo 1) que muestra en el plano apical de 5 cámaras una imagen móvil e hiperecogénica de flap en la aorta torácica ascendente que prolapsa en diástole hacia el tracto de salida del ventrículo izquierdo con insuficiencia aórtica moderada-severa concomitante, y FEVI ligera-moderadamente deprimida. Con todo ello, se establece el diagnóstico de sospecha de SAA.

El diagnóstico se confirma mediante TAC urgente (Figura 3, 4, 5) donde se comprueba la presencia de SAA tipo disección de aorta tipo A (según la clasificación de Stanford, o I de la clasificación deBakey) y se identifica un flap intimal que se extiende desde el origen de la aorta ascendente hasta la aorta torácica descendente (nivel T9), afecta al ostium del tronco de la coronaria izquierda y se prolonga cranealmente hacia el tronco braquiocefálico común derecho, sin llegar a sus ramas. Los troncos supraaórticos emergen de la luz verdadera. Se objetiva además una dilatación de la aorta torácica ascendente con un diámetro máximo de $4,9 \mathrm{~cm}$

Ante el deterioro progresivo del paciente, éste es intubado y trasladado a la Unidad de Cuidados Críticos Cardiológicos; y se consulta con Cirugía Cardíaca para intervención quirúrgica emergente. Previamente a la cirugía se realiza un ecocardiograma transesofágico (Vídeo 2, 3, 4, 5, 6) donde se visualiza el flap en aorta torácica ascendente, y destaca un rápido deterioro de la función biventricular, con FEVI ahora severamente deprimida- (20-25\%), junto a disfunción ventricular derecha severa.

Finalmente, ante el rápido empeoramiento y la situación crítica del enfermo pese a medidas de soporte avanzado, el Heart Team, decide desestimar el procedimiento quirúrgico ante el mal pronóstico a muy corto plazo, y la mortalidad extrema de la cirugía, falleciendo el paciente poco minutos después.

\section{Estudio por imagen}

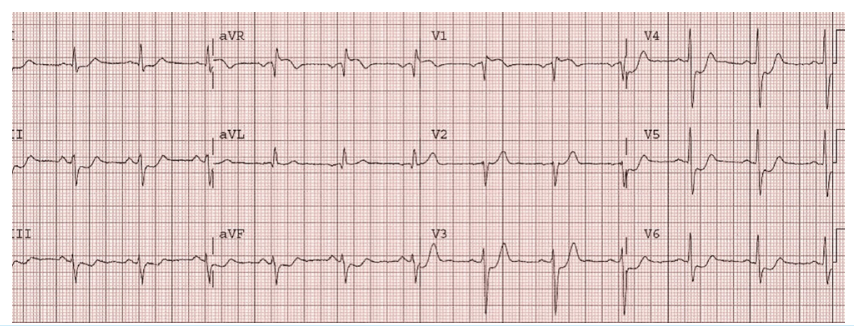

Figura 1. ECG al ingreso en Urgencias. El descenso difuso de ST con ascenso en aVR plantean la sospecha de lesión del tronco común de la coronaria izquierda

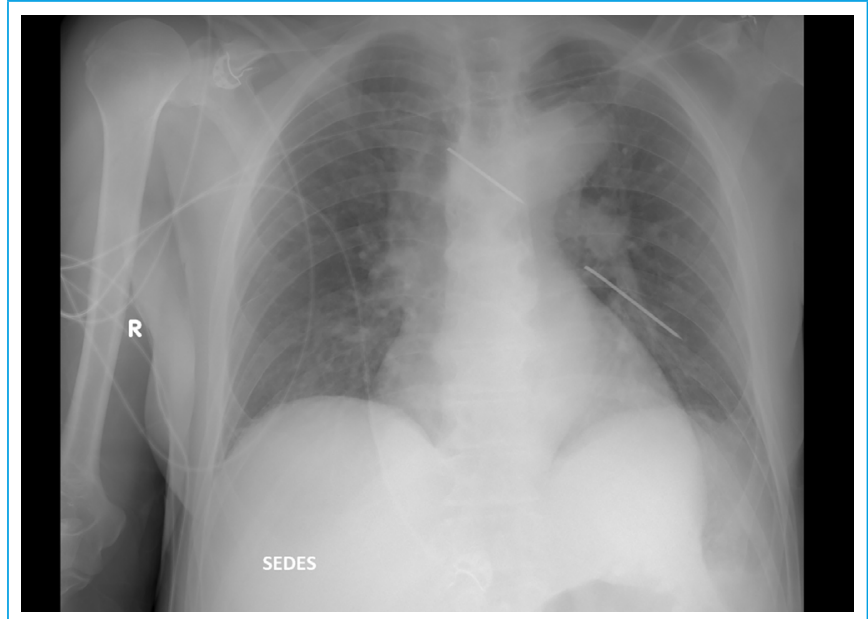

Figura 2. RX de tórax en Box de críticos. Destaca ensanchamiento marcado del mediastino superior

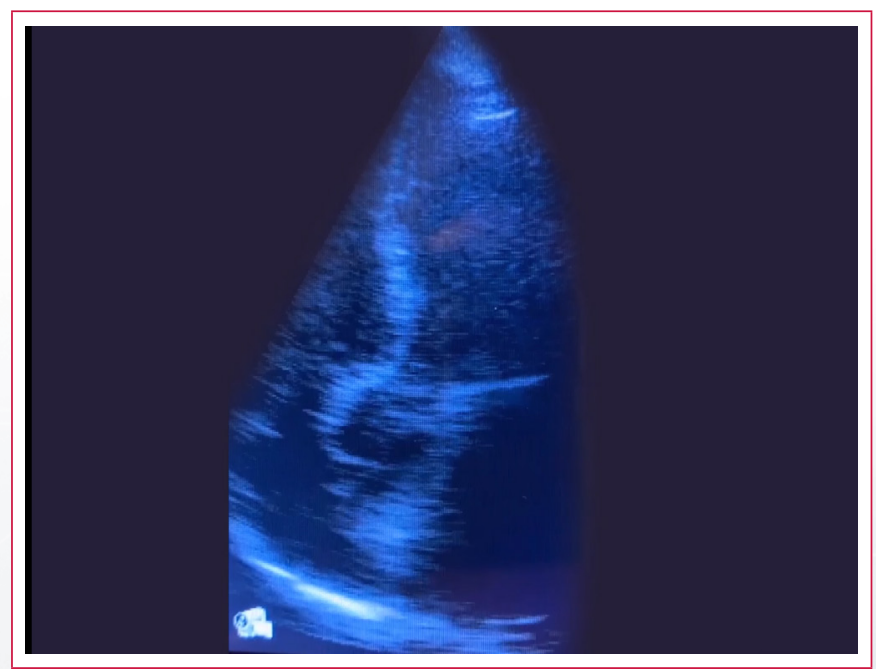

Vídeo 1. Imagen del ecocardiograma realizado en Urgencias. La forma de poder grabarla fue hacer con el móvil un video de la pantalla del equipo, por lo que la calidad es muy mala. Sin embargo es suficiente para ver un plano de 5 cámaras con un flap en aorta ascendente que prolapsa en el tracto de salida de VI compatible con disección de aorta

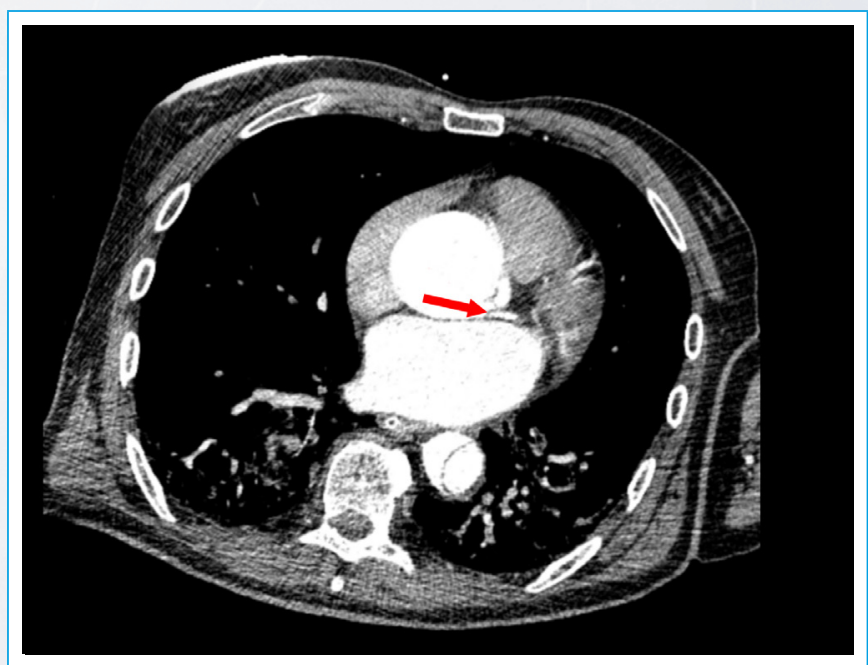

Figura 3. Estudio con TC Se aprecia un flap en aorta ascendente que compromete el origen del tronco común izquierdo 


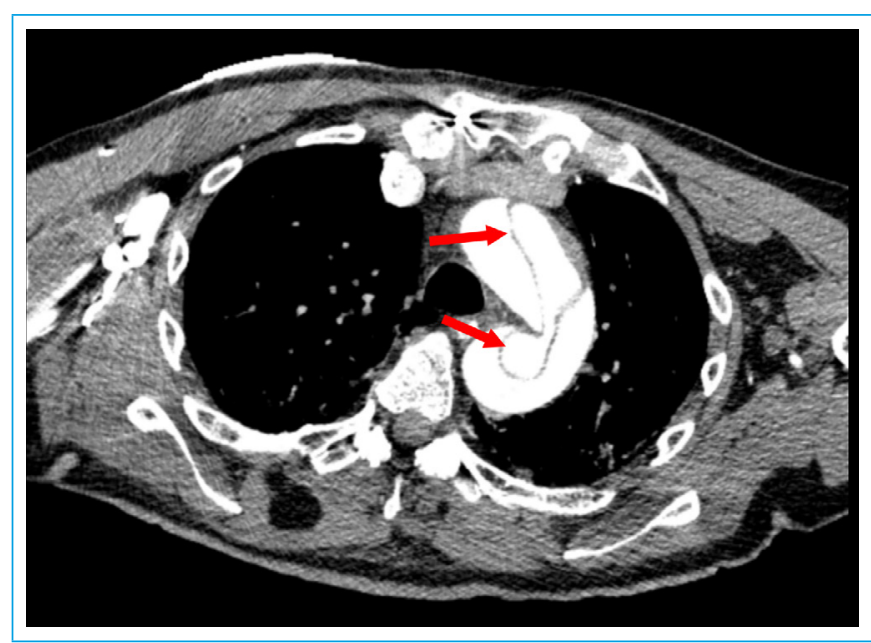

Figura 4. Estudio con TC Imagen axial que muestra el flap de disección en cayado aórtico

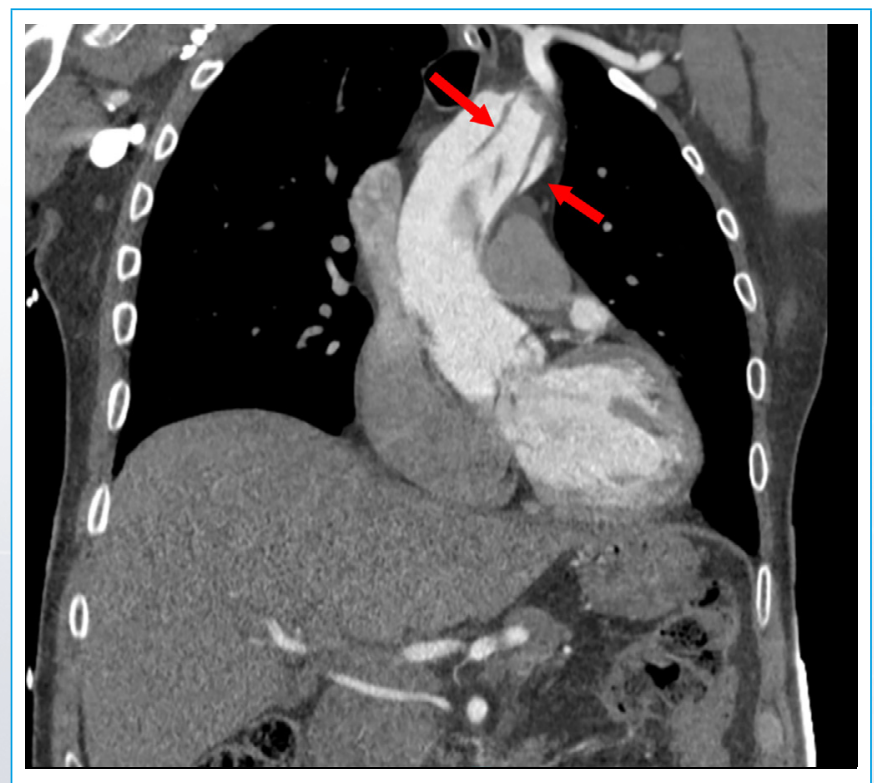

Figura 5. Estudio con TC Imagen coronal que muestra el flap de disección en aorta ascendente y cayado

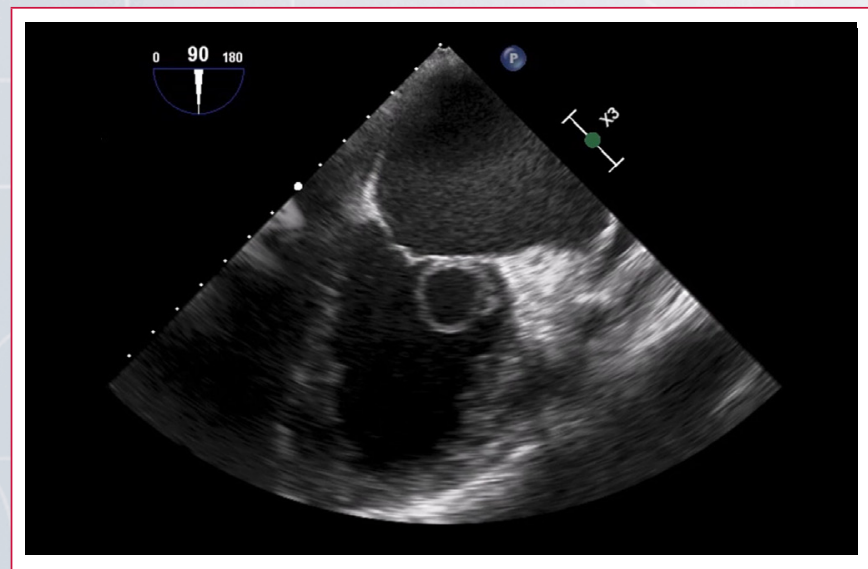

Vídeo 2. Estudio ETE a 90. Imagen de dos cámaras. Destaca disfunción ventricular severa. En la cavidad ventricular vemos aparecer y desaparecer una estructura redondeada que corresponde al flap de aorta que entra en el plano en diástole, cuando el ventrículo se hace más grande

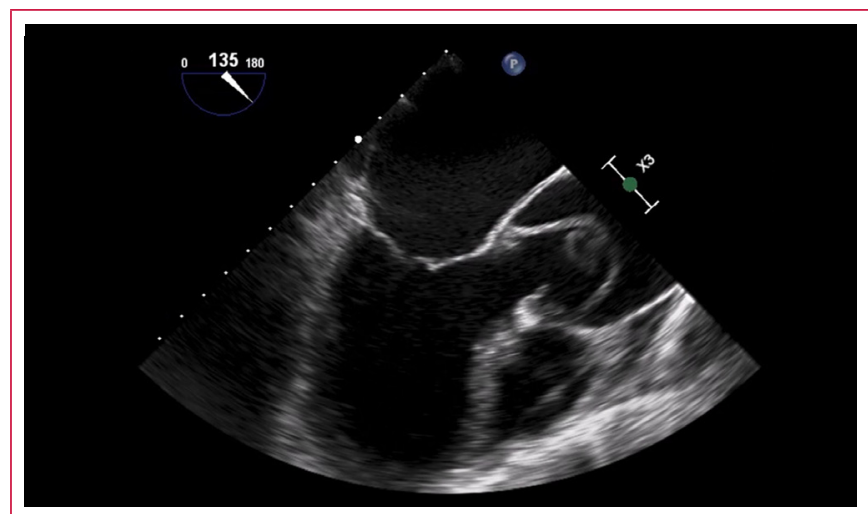

Vídeo 3. Estudio ETE a 135. Imagen de tres cámaras. Se aprecia un gran flap de aorta ascendente que prolapsa de forma amplia en tracto de salida

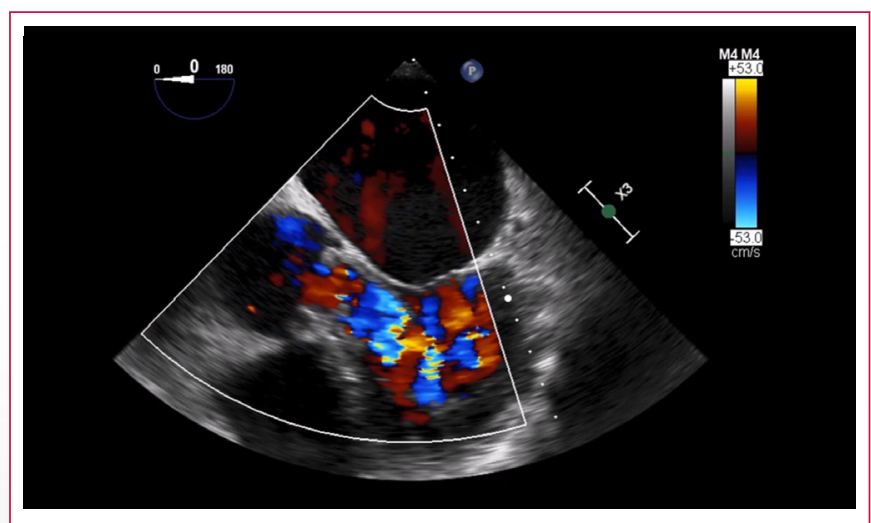

Vídeo 4 . Estudio ETE a $0^{\circ}$. En el tracto de salida de VI se aprecia un jet diastólico muy ancho y breve que corresponde a una insuficiencia severa.

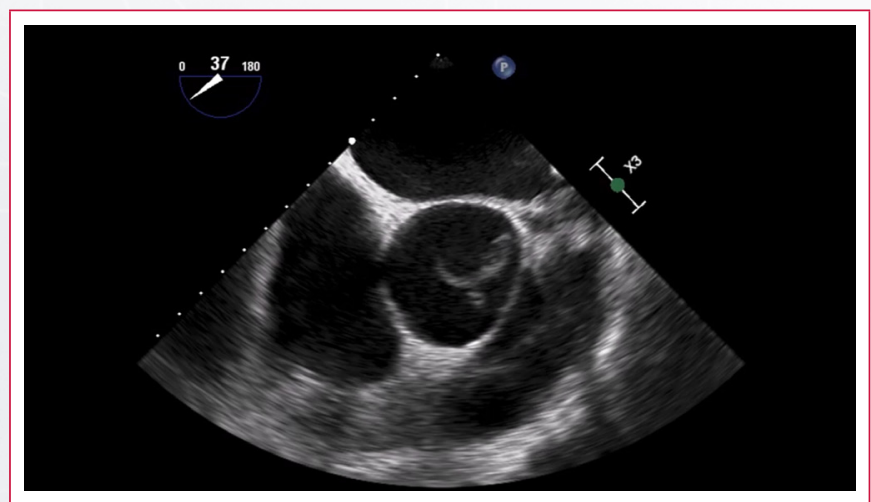

Vídeo 5. Estudio ETE a $45^{\circ}$ Imagen transversal de la raíz aórtica que demuestra disección prácticamente circunferencial de la luz

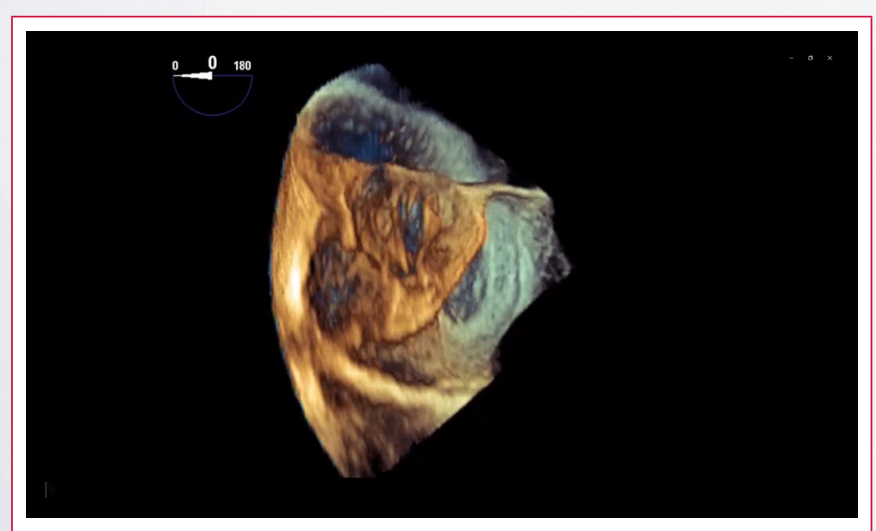

Vídeo 6. Estudio con ETE 3D de la raíz de la aorta a nivel de la imagen del video 05 


\section{Discusión}

El Síndrome Aórtico Agudo (SAA) es una situación clínica que engloba varias entidades, todas ellas caracterizadas por una presentación clínica común, que consiste en la extravasación de sangre fuera de la verdadera luz aórtica. Se incluyen tres entidades: disección aórtica aguda, hematoma intramural aórtico y la úlcera aterosclerótica penetrante ${ }^{(1)}$.

La disección aórtica aguda, la más frecuente de estas entidades, típicamente localizada en la aorta ascendente; se produce como consecuencia de un desgarro intimal, que permite el paso de sangre hacia la capa media, formando una falsa luz vascular; respetándose la capa adventicia ${ }^{(2-5)}$. El agente etiológico identificable más frecuentemente en el SAA es la hipertensión arterial (75\% de los casos); la cual se encontraba presente en nuestro paciente. No obstante, el principal factor etiopatogénico predisponente es la degeneración o necrosis quística de la capa media (típica de paciente con enfermedades del tejido conjuntivo, HTA, válvula aórtica bicúspide congénita). Aunque este hecho no estaba confirmado en nuestro paciente, la historia previa de rotura previa de un aneurisma ilíaco, podría sugerir que la pared de la aorta podría tener patología de base.

Los síntomas del SAA son muy variables, es por ello que la sospecha clínica inicial es fundamental, y debe ser el primer paso para un diagnóstico temprano, que permita una actitud terapéutica precoz. En nuestro caso, en un paciente con dolor torácico debe cumplirse la máxima de realización de un ECG en los primeros 10 minutos desde su llegada al SUH. Las alteraciones electrocardiográficas sugestivas de enfermedad del tronco común izquierdo (TCI) que presentaba nuestro paciente debe ponernos siempre en alerta de la posibilidad de compromiso a este nivel, no únicamente por una oclusión trombótica, sino también por la opción de una disección aórtica que afecte al ostium del TCl; tal y como era en nuestro caso.

La inmediata disponibilidad que existe en la actualidad en los SUH para llevar a cabo pruebas de imagen como la ecocardiografía transtorácica puede ayudarnos a orientar nuestra sospecha de forma rápida, y guiar así las siguientes pruebas de confirmación diagnóstica, tal y como se ha presentado en nuestro caso. El estudio con eco planteó rápidamente la sospecha de disección de aorta, que se confirmó con el TC. Por otro lado, la realización de un ecocardiograma transesofágico (ETE) puede ayudarnos a caracterizar la afectación de la aorta torácica y/o valvular y planificar así el procedimiento quirúrgico emergente. En nuestro paciente, el ETE confirmó la presencia del flap aórtico, con disección del ostium del TCl, así como una insuficiencia aórtica masiva.

La mortalidad de esta entidad supera el $60 \%$ en la primera semana de evolución si no se inicia el tratamiento adecuado rápidamente. La mortalidad en las primeras 24 horas se sitúa en torno al 20\%; a las 48 horas, del 38\%; a los 7 días, del 62\%, y a los 14 días, del 74\%. Pese a que el diagnóstico se estableció rápidamente en nuestro caso, finalmente el paciente falleció a las pocas horas dada la inestabilidad clínica que presentaba pese a las medidas de resucitación avanzada realizadas.

\section{Conclusión}

El Síndrome aórtico agudo, y en concreto la disección aórtica tipo A, es una entidad con una alta letalidad y con un pronóstico desolador si no se realiza una intervención quirúrgica emergente. El papel de las pruebas de imagen es fundamental, siendo la ecocardiografía y la tomografía axial computarizada los puntos clave en el diagnóstico; erigiéndose como el primer paso para una rápida actuación

\section{Ideas para recordar}

- El síndrome aórtico agudo es una etiología con una alta letalidad sin una actuación rápida y adecuada por lo que se precisa una alta sospecha diagnóstica en un paciente con un perfil compatible.

- Tanto el ecocardiograma transtorácico -en el diagnóstico de sospecha-, como la tomografía axial computarizada -para la confirmación diagnósticason las técnicas de imagen para el diagnóstico en función de la estabilidad del paciente.

- El ecocardiograma transesofágico es especialmente útil cuando se sospecha afectación de la lesión de la válvula aórtica, lo que es muy importante de cara a la planificación del procedimiento quirúrgico..

\section{Bibliografía}

1. Erbel R, et al. Guía ESC 2014 sobre diagnóstico y tratamiento de la patología de la aorta. Rev Esp Cardiol 2015; 68 (3): 242.e1-e69.

2. Evangelista A. Avances en el Síndrome Aórtico Agudo. Rev Esp Cardiol 2007; 60 (4):428-439.

3. Douglas L, Mann DPZ Peter Libby, Robert O Bonow; founding editor and online editor Eugene Braunwald. Braunwald's heart disease: a textbook of cardiovascular medicine. 10ª ed. Elsevier/Saunders Philadelphia, PA, 2015.

4. Vázquez Lima M.J, Casal Codesido J. R. Guía de actuación en Servicio de Urgencias. 4a Ed. El Bierzo; Ofelmaga. 2012.

5. Hagan P, Nienaber C, Isselbacher C, Bruckman D, Karavite D, Russman P, et al. The International Registry of Acute Aortic Dissection (IRAD). JAMA 2000; 283: 897-903. 